\title{
Una Revisión Sistemática de la Literatura Enfocada en el uso de Gamificación en Equipos de Trabajo en la Ingeniería de Software
}

\author{
Luis Hernández ${ }^{1}$, Mirna Muñoz ${ }^{1}$, Jezreel Mejía ${ }^{1}$, Adriana Peña ${ }^{2}$, Nora Rangel ${ }^{3}$, Carlos \\ Torres $^{3}$ \\ luis.hernandez@cimat.mx,mirna.munoz@cimat.mx,jmejia@cimat.mx, adriana.pena@cucei. \\ udg.mx, norarangel@cucba.udg.mx, jtorres@cencar.mx \\ ${ }^{1}$ Centro de Investigación en Matemáticas - Unidad Zacatecas, C.P. 98068, Zacatecas, Zacatecas, México. \\ ${ }^{2}$ Centro Universitario de Ciencias Exactas e Ingenierías de la Universidad de Guadalajara, Blvrd. Marcelino \\ García Barragán \#1421, esq. Calzada Olímpica, C.P. 44430, Guadalajara, Jalisco, México. \\ ${ }^{3}$ Centro de Estudios e Investigaciones en Comportamiento de la Universidad de Guadalajara, Calle Francisco \\ de Quevedo \# 18o, Col. Arcos Vallarta, C.P. 44130, Guadalajara, Jalisco, México
}

DOI: 10.17013/risti.21.33-50

\begin{abstract}
Resumen: Actualmente la gamificación ha sido aplicada más allá de los juegos tradicionales que conocemos, por ejemplo, ha sido aplicada en la educación de temas en la ingeniería de software, donde apoya el aprendizaje y cumplimiento de actividades en los proyectos. Sin embargo, existe muy poca evidencia de su aplicación para mejorar la integración de un equipo, donde existen diferentes factores que intervienen en su desempeño. Uno de los factores más importantes es el trabajo colaborativo, habilidad que todo ingeniero de software debe tener. Basado en lo anterior, la integración adecuada de un equipo influye en el rendimiento del mismo, por esto se deben complementar las habilidades, conocimientos y estilos interactivos de cada miembro para obtener un equipo altamente efectivo. Este estudio presenta los resultados de una revisión sistemática, enfocada en el uso de gamificación para crear un equipo, reduciendo el tiempo de integración y por lo tanto mejorar su rendimiento.
\end{abstract}

Palabras-clave: Gamificación; equipos de trabajo; trabajo colaborativo; elementos de gamificación; ingeniería de software; revisión sistemática de la literatura.

\section{A systematic literature review focused on the use of gamification in software engineering teamworks}

\begin{abstract}
Nowadays, the gamification has been applied beyond of traditional games that we know, an example is their application in topics in software engineering education, where it supports the learning and the accomplishment of activities in projects. However, there is very few evidence of its implementation to improve the integration of team work members, where there are several factors involved in their performance. One of the most important factor is the collaborative work,
\end{abstract}


fundamental skill that every software engineer should have. Based on the above mentioned, the adequate integration of a teamwork influences its performance. Therefore, the skills, knowledge and interactive style for each member it should be complemented, in order to get a high effective team. This study presents results of a systematic literature review focused in gamification uses to create a team, reducing its integration time and therefore, improve its performance.

Keywords: Gamification; teamwork; collaborative work; gamification elements; software engineering; systematic literature review.

\section{Introducción}

En los últimos años las técnicas de gamificación han sido aplicadas más allá del dominio de los juegos tradicionales que conocemos, tal es el caso en el área de la educación, salud, mercadotecnia e ingeniería de software (Estacio et al., 2014; Jurado, Fernandez, \& Collazos, 2015).

Dentro de la ingeniería de software se han originado oportunidades para utilizar la gamificación en el desarrollo de productos, así como en la mejora de procesos de software (E. Hernández, Colomo Palacios, \& de Amescua Seco, 2010; Osborne O’Hagan, Coleman, \& O'Connor, 2014). De hecho, de acuerdo a la investigación de M2 Advisory Group (M2 Research, 2016), se estima que en el año 2016 el tamaño del mercado con respecto al uso de gamificación logrará más de 2.8 millones de dólares, tan sólo en Estados Unidos.

En este contexto, no se sabe con exactitud si existen técnicas para crear un equipo de trabajo utilizando técnicas de gamificación, aunque se puede lograr la identificación de perfiles de los miembros del equipo de desarrollo mediante su uso (Jurado et al., 2015; Vasconcelos et al., 2017).

Este estudio tiene como objetivo identificar los elementos de gamificación que actualmente están siendo aplicados en equipos de trabajo, principalmente en el área de ingeniería de software. Para esto, se presenta una Revisión Sistemática de la Literatura (RSL) para identificar cuáles son las técnicas de gamificación aplicadas en el trabajo en equipo, la identificación de perfiles de trabajo y la mejora de su rendimiento en la ingeniería de software. Los resultados de la RSL serán de apoyo para la creación de equipos de trabajo altamente efectivos.

El estudio está organizado de la siguiente forma: en la sección 2 se describen los antecedentes que fueron utilizados para llevar a cabo este artículo. La Sección 3 presenta los trabajos relacionados con este estudio. La Sección 4 describe el protocolo usado en la RSL. En la sección 5 se presenta el análisis de los resultados de la RSL. Finalmente, la sección 6 presenta la conclusión y trabajo futuro.

\section{Antecedentes}

El término de gamificación se refiere, de manera general, a la aplicación de elementos de juego en contexto que no son de juego, mejorando la experiencia y compromiso de los participantes (Korn, Funk, \& Schmidt, 2015b). 
La aplicación de gamificación en entornos de educación, específicamente en el área de ingeniería de software (Berkling, 2016) es considerada como una herramienta viable, porque permite animar a los estudiantes a participar e interactuar efectivamente en proyectos realizados en el salón de clases (Akpolat \& Slany, 2014). También ha logrado la satisfacción de los estudiantes durante su participación (Iosup \& Epema, 2014) ocasionando que disfruten el aprendizaje y de esta forma se conviertan en estudiantes más competitivos (Lynch et al., 2011). Asimismo se ha encontrado un artículo que hace uso de gamificación con realidad virtual para promover y mejorar el trabajo en equipo (Gasca-Hurtado, Peña, Gómez-Álvarez, Plascencia-Osuna, \& Calvo-Manzano, 2015).

Por otro lado, en el entorno industrial se han presentado iniciativas para mejorar las interacciones sociales (Stanculescu, Bozzon, Sips, \& Houben, 2016) y mejorar la comunicación del conocimiento de los trabajadores (Jurado et al., 2015), por mencionar algunos ejemplos: 1) una aplicación web, 2) una plataforma de preguntas y respuestas, y 3) un centro de noticias.

En la ingeniería de software el trabajo es realizado en equipos, por lo tanto, dentro del conjunto de soft skills deseadas por parte de un ingeniero de software la capacidad para trabajar en equipo es esencial (J. Chen, Qiu, Yuan, Zhang, \& Lu, 2011). Así como otras habilidades catalogadas tanto para los líderes como miembros de un equipo, por mencionar algunas de las más importantes: 1) liderazgo, 2) habilidad de comunicación, 3) analítico y 4) habilidad para la resolución de problemas (Matturro, Raschetti, \& Fontán, 2015).

Al día de hoy se pueden encontrar propuestas para mejorar las áreas de desarrollo de software, en (Kosa \& Yilmaz, 2010) muestran una serie de juegos que pueden ser usados para mejorar los procesos de desarrollo de software, por ejemplo: un juego de cartas para cursos de ingeniería de software, un video juego enfocado en temas de seguridad o un juego para mejorar la habilidad en la toma de decisiones. Por otro lado, la tarea de realizar revisiones de código es tan importante como la propia codificación, esta iniciativa es presentada en (Unkelos-Shpigel \& Hadar, 2015), donde se propone aplicar gamificación a dicha actividad para ser realizada con un fuerte compromiso por parte de los inspectores de código.

\section{Trabajos relacionados}

Para el desarrollo de este estudio se han identificado cuatro trabajos relacionados, debido a la aportación de conocimiento relevante referente a los temas de gamificación y equipos de trabajo. Estos estudios permiten ampliar y conocer el estado de los temas hasta el momento y desde otro punto de vista. A continuación, en la Tabla 1 se describen los estudios. 


\begin{tabular}{|c|c|c|c|c|}
\hline Estudio & Propuesta & Contexto & Objetivo & Justificación \\
\hline $\begin{array}{l}\text { (Guenaga, } \\
\text { Eguiluz, } \\
\text { Rayon, } \\
\text { Nunez, \& } \\
\text { Quevedo, } \\
\text { 2014) }\end{array}$ & $\begin{array}{l}\text { Juego serio } \\
\text { para evaluar } \\
\text { competencias } \\
\text { del equipo }\end{array}$ & Negocios & $\begin{array}{l}\text { Demostrar las } \\
\text { consecuencias } \\
\text { en la toma } \\
\text { de decisiones } \\
\text { para mejorar la } \\
\text { comunicación } \\
\text { entre los } \\
\text { miembros del } \\
\text { equipo }\end{array}$ & $\begin{array}{l}\text { Evalúa el trabajo en equipo con un } \\
\text { juego serio apoyado con los elementos } \\
\text { de gamificación, por lo que aporta } \\
\text { información relevante para conocer } \\
\text { cómo impactan los elementos de } \\
\text { gamificación en el equipo }\end{array}$ \\
\hline $\begin{array}{l}\text { (E. } \\
\text { Hernández } \\
\text { et al., 2010) }\end{array}$ & $\begin{array}{l}\text { Plataforma } \\
\text { web para } \\
\text { incrementar la } \\
\text { motivación en } \\
\text { los proyectos de } \\
\text { software }\end{array}$ & $\begin{array}{l}\text { Mejora de } \\
\text { procesos de } \\
\text { software en } \\
\text { el desarrollo }\end{array}$ & $\begin{array}{l}\text { Incrementar la } \\
\text { motivación de } \\
\text { los miembros } \\
\text { del equipo para } \\
\text { participar en } \\
\text { la mejora de } \\
\text { procesos de } \\
\text { software }\end{array}$ & $\begin{array}{l}\text { Enfoca los equipos de trabajo en el } \\
\text { área de la mejora de procesos para } \\
\text { el desarrollo de software, aportando } \\
\text { información clave en base al } \\
\text { desempeño del equipo con respecto al } \\
\text { uso de una plataforma web con apoyo } \\
\text { de los elementos de gamificación }\end{array}$ \\
\hline $\begin{array}{l}\text { (Latulipe, } \\
\text { Long, \& } \\
\text { Seminario, } \\
\text { 2015) }\end{array}$ & $\begin{array}{l}\text { Enfoque } \\
\text { para ayudar } \\
\text { a las clases } \\
\text { de ciencias } \\
\text { computacionales } \\
\text { a ser más } \\
\text { sociales y } \\
\text { efectivas }\end{array}$ & $\begin{array}{l}\text { Educación } \\
\text { en el área de } \\
\text { ciencias de la } \\
\text { computación }\end{array}$ & $\begin{array}{l}\text { Formar equipos } \\
\text { ligeros para } \\
\text { mejorar el } \\
\text { aprendizaje de } \\
\text { los estudiantes } \\
\text { en cursos de } \\
\text { programación }\end{array}$ & $\begin{array}{l}\text { Enfoca el trabajo de equipo en el } \\
\text { área académica para aprovechar los } \\
\text { beneficios del aprendizaje basado en } \\
\text { equipo y eliminar el estrés asociado } \\
\text { con los riesgos en el desarrollo de } \\
\text { proyectos }\end{array}$ \\
\hline $\begin{array}{l}\text { (Dutra, } \\
\text { Prikladnicki, } \\
\text { \& Franca, } \\
\text { 2015) }\end{array}$ & $\begin{array}{l}\text { Identificación de } \\
\text { características } \\
\text { que influyen } \\
\text { positivamente y } \\
\text { negativamente } \\
\text { en el desempeño } \\
\text { de un equipo de } \\
\text { trabajo }\end{array}$ & $\begin{array}{l}\text { Equipos en } \\
\text { la ingeniería } \\
\text { de software }\end{array}$ & $\begin{array}{l}\text { Entender } \\
\text { cuáles son los } \\
\text { contextos y las } \\
\text { condiciones } \\
\text { en las cuales } \\
\text { los equipos en } \\
\text { la ingeniería } \\
\text { de software } \\
\text { trabajan }\end{array}$ & $\begin{array}{l}\text { Aporta un panorama general acerca } \\
\text { factores a tener en cuenta en un } \\
\text { equipo en el área de la ingeniería } \\
\text { de software, y de esta manera se } \\
\text { pueda convertir en un equipo de alto } \\
\text { desempeño }\end{array}$ \\
\hline $\begin{array}{l}\text { (Knutas, } \\
\text { Ikonen, } \\
\text { Nikula, } \\
\text { \& Porras, } \\
\text { 2014) }\end{array}$ & $\begin{array}{l}\text { Organizar un } \\
\text { modelo de } \\
\text { gamificación } \\
\text { en torno a la } \\
\text { colaboración de } \\
\text { los estudiantes }\end{array}$ & $\begin{array}{l}\text { Educación } \\
\text { en el área de } \\
\text { ciencias de la } \\
\text { computación }\end{array}$ & $\begin{array}{l}\text { Mejorar la } \\
\text { comunicación } \\
\text { de los } \\
\text { estudiantes } \\
\text { por medio de } \\
\text { un modelo de } \\
\text { gamificación }\end{array}$ & $\begin{array}{l}\text { Analiza como los elementos de } \\
\text { gamificación pueden incrementar } \\
\text { la comunicación en los equipos } \\
\text { académicos }\end{array}$ \\
\hline $\begin{array}{l}\text { (Poy-Castro, } \\
\text { Mendaña- } \\
\text { Cuervo, \& } \\
\text { González, } \\
\text { 2015) }\end{array}$ & $\begin{array}{l}\text { Crear un juego } \\
\text { serio para } \\
\text { fomentar las } \\
\text { habilidades } \\
\text { de trabajo en } \\
\text { equipo en los } \\
\text { estudiantes } \\
\text { universitarios }\end{array}$ & $\begin{array}{l}\text { Estudiantes } \\
\text { del grado en } \\
\text { Educación } \\
\text { Infantil }\end{array}$ & $\begin{array}{l}\text { Poner a los } \\
\text { estudiantes en } \\
\text { una situación } \\
\text { de estrés } \\
\text { que origine } \\
\text { la necesidad } \\
\text { de trabajo en } \\
\text { equipo para } \\
\text { resolver el } \\
\text { problema }\end{array}$ & $\begin{array}{l}\text { Demuestra como el desarrollo de un } \\
\text { juego serio motiva a los estudiantes } \\
\text { para participar en actividades } \\
\text { colaborativas y de esta manera } \\
\text { fomentar el trabajo en equipo gracias } \\
\text { a la motivación que proporciona un } \\
\text { juego serio }\end{array}$ \\
\hline
\end{tabular}

Tabla 1 - Trabajos Relacionados 


\section{Método de investigación}

Este artículo es una versión extendida del trabajo publicado en (L. Hernández, Muñoz, Mejia, \& Peña, 2016), el cual se desarrolló en base al protocolo formal de investigación de la revisión sistemática de la literatura (RSL), propuesto por B. Kitchenham (Kitchenham \& Charters, 2007), el cual se aborda abordando las fases de: 1) Planificación de la revisión, 2) Ejecución de la revisión y 3) Reporte de resultados. En esta sección muestra parte de la extensión del trabajo previo.

\subsection{Planificación de la Revisión}

La primera fase consiste en la planificación de la RSL donde se tiene en cuenta los siguientes pasos: identificación de la necesidad de la RSL, especificación de las preguntas de investigación, creación de la cadena de búsqueda y finalmente la identificación de las fuentes de investigación, los pasos anteriores pueden ser consultados de forma completa en (L. Hernández et al., 2016). Las preguntas de investigación se describen a continuación en la Tabla 2.

\begin{tabular}{lll}
\hline ID & Pregunta & Objetivo \\
\hline PI1 & $\begin{array}{l}\text { ¿Qué elementos de gamificación han sido } \\
\text { aplicados para la creación de equipos de } \\
\text { trabajo y cuáles han sido más efectivos? }\end{array}$ & $\begin{array}{l}\text { Identificar los elementos de gamificación que han } \\
\text { sido empleados para la creación de un equipo de } \\
\text { trabajo y determinar cuáles han logrado mejores } \\
\text { resultados de su aplicación. }\end{array}$ \\
\hline PI2 & $\begin{array}{l}\text { ¿En cuáles entornos han sido aplicadas las } \\
\text { ténicas de gamificación para la creación de } \\
\text { equipos? }\end{array}$ & $\begin{array}{l}\text { Descubrir los entornos donde han aplicados técnicas } \\
\text { de gamificación para la creación y trabajo en equipo. }\end{array}$ \\
\hline PI3 & $\begin{array}{l}\text { ¿Cuáles elementos de gamificación han } \\
\text { sido aplicadas para mejorar el trabajo en } \\
\text { equipo? }\end{array}$ & $\begin{array}{l}\text { Descubrir los elementos de gamificación que apoyan } \\
\text { la mejora del trabajo en equipo. }\end{array}$ \\
\hline PI4 & $\begin{array}{l}\text { ¿Cuáles han sido las principales } \\
\text { implementaciones o herramientas para } \\
\text { aplicar los elementos de gamificación? }\end{array}$ & $\begin{array}{l}\text { Identificar las implementaciones o herramientas que } \\
\text { han sido empleadas para llevar a cabo de las técnicas } \\
\text { de gamificación en los equipos de trabajo. }\end{array}$ \\
\hline Pi5 & $\begin{array}{l}\text { ¿Cuáles son los objetivos a cumplir con el } \\
\text { apoyo de los elementos de gamificación? }\end{array}$ & $\begin{array}{l}\text { Reconocer los objetivos que persiguen los estudios } \\
\text { mediante la aplicación de los elementos. }\end{array}$ \\
\hline
\end{tabular}

Tabla 2 - Preguntas de investigación

Para la creación de la cadena de búsqueda se identificaron las palabras clave basadas en las preguntas de investigación descritas en la Tabla 2 siendo: gamification, team, teams, teamwork y software engineering. Posteriormente se combinaron las palabras clave y mediante el uso de los operadores lógicos AND y OR, resultando en la siguiente cadena de búsqueda general: (Gamification) AND (Team OR Teams OR Teamwork) $A N D$ (Software engineering).

\subsection{Ejecución de la revisión}

Durante la segunda fase de la revisión la actividad principal es la recolección de estudios, el resultado es un conjunto de estudios primarios para la investigación. La aplicación de 
la cadena de búsqueda se realizó durante en el periodo del 4 al 11 de mayo del año 2016 en cada una de las bibliotecas digitales.

La selección de Estudios Primarios (EP) fue realizada en cuatro pasos: 1) ejecución de la cadena de búsqueda para cada una de las bibliotecas digitales, 2) aplicación de criterios de inclusión y criterios de exclusión (ver Tabla 3), si el estudio estaba relacionado de manera indirecta se procedía a incluirlo para el siguiente paso; 3) lectura del título y resumen para identificación de estudios candidatos a ser elegidos como EP, en caso de ser necesario se realizó la lectura de la introducción y la conclusión; y 4) selección de EP para este artículo.

\begin{tabular}{ll}
\hline Criterios de inclusión & Criterios de exclusión \\
\hline $\begin{array}{l}\text { Estudios donde el título contenga por lo menos } \\
\text { dos palabras clave de la cadena de búsqueda, } \\
\text { principalmente Gamificación. }\end{array}$ & $\begin{array}{l}\text { Estudios donde el título no contenga las palabras } \\
\text { clave de la cadena de búsqueda. }\end{array}$ \\
\hline $\begin{array}{l}\text { Estudios donde el resumen presente una relación } \\
\begin{array}{l}\text { con la aplicación de técnicas o elementos de } \\
\text { gamificación en equipos de trabajo. }\end{array}\end{array}$ & $\begin{array}{l}\text { Estudios donde el resumen no esté relacionado con } \\
\text { la aplicación de técnicas de gamificación. }\end{array}$ \\
\hline $\begin{array}{l}\text { Estudios con el contexto principal enfocado en } \\
\text { ingeniería de software o trabajo en equipo. }\end{array}$ & $\begin{array}{l}\text { Estudios que no aborden el tema de equipos de } \\
\text { trabajo. }\end{array}$ \\
\hline Estudios que estén escritos en inglés. & Estudios que no estén en inglés. \\
\hline Estudios publicados entre los años: 2010 - 2016. & Estudios publicados antes del año 2010. \\
\cline { 2 - 2 } & Estudios duplicados. \\
\hline
\end{tabular}

Tabla 3 - Criterios de inclusión y exclusión

Durante el seguimiento de los pasos se obtuvieron los siguientes resultados: de un total de 2,214 existieron cuatro estudios duplicados y finalmente se obtuvieron $31 \mathrm{EP}$, en la Tabla 4 se detalla el resumen del proceso de selección.

\begin{tabular}{lllll}
\hline Pasos & $\begin{array}{l}\text { ACM } \\
\text { DL }\end{array}$ & $\begin{array}{l}\text { IEEE } \\
\text { Eplore }\end{array}$ & $\begin{array}{l}\text { Springer } \\
\text { Link }\end{array}$ & Total \\
\hline Paso 1 - Ejecución de la cadena de búsqueda & 132 & 1761 & 321 & 2214 \\
\hline Paso 2 - Aplicación de Criterios de inclusión y exclusión & 26 & 36 & 8 & 80 \\
\hline Paso 3 - Lectura Título y Resumen & 17 & 12 & 2 & 32 \\
\hline Paso 4 - Selección de estudios primarios. & 17 & 12 & 1 & 31 \\
\hline
\end{tabular}

Tabla 4 - Proceso de selección de EP

Finalmente se aplicaron un conjunto de preguntas con el objetivo de evaluar la calidad (AC) en los estudios seleccionados: AC1) ¿El estudio se enfoca en la aplicación de técnicas de gamificación en equipos de trabajo?, AC2) ¿El estudio aborda la relación 
entre el trabajo en equipo y su rendimiento? Y AC3 ¿El estudio aborda la relación entre los elementos de gamificación y el rendimiento del equipo?

La evaluación de los criterios para el aseguramiento de la calidad de los EP se llevó a cabo seleccionando aquellos estudios que por lo menos cumplieran con una de las preguntas del AC.

El resumen de los EP seleccionados para este estudio se detalla en la Tabla 5, donde se especifica un identificador (ID), su Referencia y el cumplimiento en base a los criterios para el aseguramiento de la calidad que fueron contemplados en los EP. Donde se calificaron de la siguiente forma: 1) • estudio que cumplió completamente el criterio (100\%), 2) ๑ estudio que cumplió de forma parcial el criterio (50\%), 3) • estudio que cumplió de forma eventual el criterio (25\%) y 4) estudio que no cumplió el criterio (o\%).

\begin{tabular}{|c|c|c|c|c|c|c|c|c|c|}
\hline ID & Referencia & AC1 & AC2 & $\mathbf{A C}_{3}$ & ID & Referencia & AC1 & AC2 & AC3 \\
\hline$E P 1$ & (Jurado et al., 2015) & $\bullet$ & $\theta$ & 0 & $E P 17$ & (Iosup \& Epema, 2014) & $\bullet$ & $\theta$ & 0 \\
\hline$E P 2$ & (Estacio et al., 2014) & $\boldsymbol{\theta}$ & 0 & O & EP18 & (Lynch et al., 2011) & 0 & $\bullet$ & 0 \\
\hline$E P_{3}$ & $\begin{array}{l}\text { (E. Hernández et } \\
\text { al., 2010) }\end{array}$ & $\bullet$ & $\boldsymbol{\theta}$ & O & EP19 & (Matturro et al., 2015) & O & $\bullet$ & O \\
\hline$E P 4$ & $\begin{array}{l}\text { (Osborne O'Hagan } \\
\text { et al., 2014) }\end{array}$ & $\bullet$ & 0 & O & $E P 2 O$ & (Kosa \& Yilmaz, 2010) & $\bullet$ & O & O \\
\hline$E P_{5}$ & $\begin{array}{l}\text { (Latulipe et al., } \\
\text { 2015) }\end{array}$ & $\boldsymbol{\theta}$ & $\boldsymbol{\theta}$ & $\bullet$ & $E P 21$ & $\begin{array}{l}\text { (Unkelos-Shpigel \& } \\
\text { Hadar, 2015) }\end{array}$ & $\boldsymbol{\theta}$ & O & $\bullet$ \\
\hline EP6 & $\begin{array}{l}\text { (Dubois \& } \\
\text { Tamburrelli, 2013) }\end{array}$ & $\bullet$ & O & O & $E P 22$ & (Guenaga et al., 2014) & $\bullet$ & $\boldsymbol{\theta}$ & $\bullet$ \\
\hline$E P 7$ & $\begin{array}{l}\text { (Stanculescu et al., } \\
\text { 2016) }\end{array}$ & $\bullet$ & O & O & $E P 23$ & $\begin{array}{l}\text { (Dorling \& McCaffery, } \\
\text { 2012) }\end{array}$ & $\bullet$ & O & O \\
\hline EP8 & $\begin{array}{l}\text { (Korn, Funk, \& } \\
\text { Schmidt, 2015a) }\end{array}$ & $\bullet$ & O & O & $E P 24$ & (H. Gaynor, 2015) & O & $\bullet$ & O \\
\hline EP9 & $\begin{array}{l}\text { (Bartel, Figas, \& } \\
\text { Hagel, 2015) }\end{array}$ & $\bullet$ & O & O & $E P 25$ & $\begin{array}{l}\text { (P. C. Chen, Chern, \& } \\
\text { Chen, 2012) }\end{array}$ & O & $\bullet$ & O \\
\hline EP1O & (Korn et al., 2015b) & $\bullet$ & O & O & $E P 26$ & (Bei, 2013) & O & $\bullet$ & O \\
\hline$E P 11$ & (Dutra et al., 2015) & O & $\bullet$ & O & $E P 27$ & $\begin{array}{l}\text { (De O. Melo, Santana, \& } \\
\text { Kon, 2012) }\end{array}$ & O & $\bullet$ & 0 \\
\hline$E P 12$ & (J. Chen et al., 2011) & O & $\bullet$ & O & $E P 28$ & $\begin{array}{l}\text { (Jovanovic, Mesquida, } \\
\text { \& Antònia, 2010) }\end{array}$ & $\bullet$ & 0 & $\boldsymbol{0}$ \\
\hline$E P 13$ & $\begin{array}{l}\text { (Teh, Baniassad, } \\
\text { Van Rooy, \& } \\
\text { Boughton, 2012) }\end{array}$ & O & $\bullet$ & 0 & $E P 29$ & $\begin{array}{l}\text { (Knutas, Ikonen, } \\
\text { Maggiorini, Ripamonti, } \\
\text { \& Porras, 2014) }\end{array}$ & $\bullet$ & $\boldsymbol{\theta}$ & 0 \\
\hline EP14 & $\begin{array}{l}\text { (Knutas, Ikonen, } \\
\text { Nikula, et al., 2014) }\end{array}$ & $\bullet$ & $\boldsymbol{\theta}$ & 0 & $E P_{3 O}$ & $\begin{array}{l}\text { (O’Donovan, Gain, \& } \\
\text { Marais, 2013) }\end{array}$ & $\bullet$ & $\boldsymbol{\theta}$ & 0 \\
\hline EP15 & (Berkling, 2016) & $\boldsymbol{\theta}$ & $\boldsymbol{\theta}$ & $\bullet$ & $E P_{31}$ & $\begin{array}{l}\text { (Buisman \& van } \\
\text { Eekelen, 2014) }\end{array}$ & $\theta$ & $\boldsymbol{\theta}$ & $\bullet$ \\
\hline EP16 & $\begin{array}{l}\text { (Akpolat \& Slany, } \\
\text { 2014) }\end{array}$ & $\boldsymbol{\theta}$ & $\boldsymbol{\theta}$ & $\bullet$ & - & - & - & - & - \\
\hline
\end{tabular}

Tabla 5 - Lista de Estudios Primarios 


\section{Resultados de la Revisión Sistemática}

En esta sección se muestran los resultados obtenidos de la RSL, los cuales permiten conocer el panorama de los elementos de gamificación aplicados en equipos de trabajo. Los hallazgos se dividen en las seis subsecciones: PI1, PI2, PI3, PI4, PI5 y resultados adicionales.

\subsection{PI1: ¿Cuáles elementos de gamificación han sido aplicados para la creación de equipos de trabajo y cuáles han sido más efectivos?}

De los EP elegidos se identificaron los principales elementos de gamificación que han sido empleados, los principales elementos se presentan en la Figura 1, la tabla de posiciones (Leaderboard en inglés) ocupa el primer lugar de aplicación y ha demostrado en (Stanculescu et al., 2016) ser un elemento útil para mejorar la participación, por otro lado, en (Latulipe et al., 2015) lograron una influencia positiva en los estudiantes obteniendo como resultado. En segundo lugar, el sistema de puntos (Points systems o Points en inglés) logró resultados positivos, por ejemplo, en (Buisman \& van Eekelen, 2014) incrementaron la participación, el compromiso y la motivación de los estudiantes. Dentro de los estudios (Latulipe et al., 2015; Stanculescu et al., 2016) las insignias (Badges en inglés) demostraron resultados positivos en combinación con la tabla de posiciones, ocupando la tercera posición.

La Figura 2, presenta el uso de elementos con menor frecuencia en los EP, sin embargo, a pesar de ser poco usuales han demostrado también ser de apoyo en combinación de otros elementos.

De manera general, la combinación de dos o más elementos de gamificación puede originar buenos resultados. Sin embargo, el éxito del resultado esperado está sujeto al entorno de la aplicación.

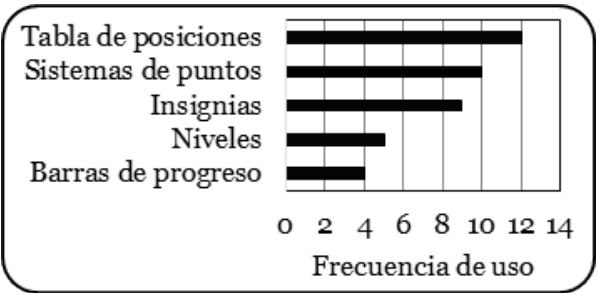

Figura 1 - Distribución de los elementos de gamificación de mayor frecuencia de uso

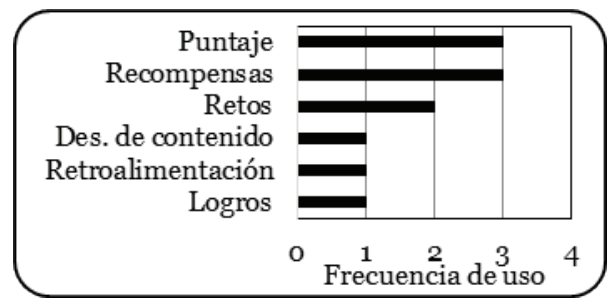

Figura 2 - Distribución de los elementos de gamificación de menor frecuencia de uso 
Es importante resaltar que la evidencia apunta sólo a los elementos que apoyan el trabajo colaborativo y el cumplimiento de las actividades asignadas, pero aún no han sido aplicados para la creación de un equipo o para determinar perfiles de trabajo.

5.2. PI2: ¿En cuáles entornos han sido aplicadas las técnicas de gamificación para la creación de equipos?

Los resultados de la distribución de los EP fueron catalogados en tres principales entornos detallados en la Figura.3: 1) académico, aquellos que están relacionados con investigaciones teóricas 2) educación, aquellas investigaciones aplicadas en el aprendizaje o casos de estudio y 3) industria, aquellos que están relacionados a investigaciones en negocios.

Se observa como los estudios académicos son los que más han explorado los temas de gamificación y equipos, por otro lado, los estudios se han enfocado más en estudiar entornos de educación que entornos de la industria. Los estudios fueron subcategorizados a partir de los entornos mencionados en la Figura4 para identificar los principales temas explorados en cada entorno.

La Figura 4 presenta los temas dentro del marco académico, se observa que las principales investigaciones son realizadas en temas de equipos (Bei, 2013; P. C. Chen et al., 2012; H. Gaynor, 2015) y gamificación (De O. Melo et al., 2012; Jovanovic et al., 2010), seguido por temas de desarrollo enfocados en el área de la ingeniería de software, identificando una baja presencia de estudios enfocados en la industria para las investigaciones académicas, identificado sólo en (De O. Melo et al., 2012).

La Figura 5 muestra la distribución de los EP enfocados en la educación, los principales EP llevan a cabo la creación de un curso para el apoyo al aprendizaje de los estudiantes, ya sea por medio de la aplicación de técnicas en un curso tradicional (Buisman \& van

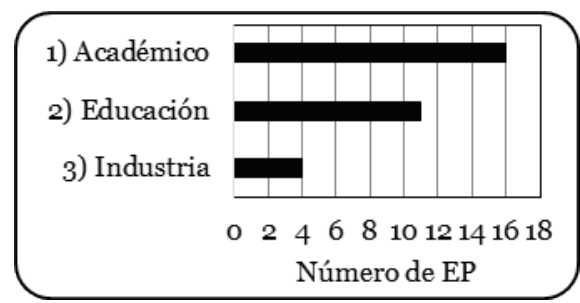

Figura 3 - Distribución de EP por entorno

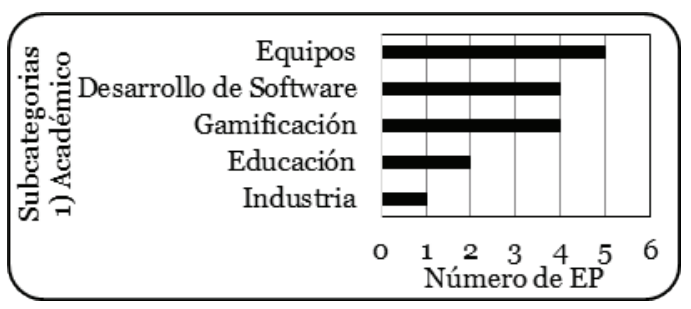

Figura 4 - Distribución de investigaciones académicas 
Eekelen, 2014; Knutas, Ikonen, Maggiorini, et al., 2014) o con el apoyo de un Learning Management System (LMS) (O’Donovan et al., 2013). Por otro lado, existe un estudio enfocado en la evaluación del trabajo en equipo por medio de un juego serio presentado en (Guenaga et al., 2014).

Finalmente, en la Figura 6 se muestra la distribución de los EP en el entorno de la industrial, los cuales se categorizaron en base a su aplicación. En el área de producción se encuentran 2 estudios (Korn et al., 2015a, 2015b); en la aplicación de negocios (Stanculescu et al., 2016) y el desarrollo de software (Jurado et al., 2015) sólo un estudio.

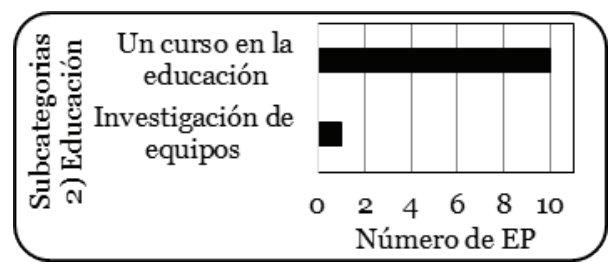

Figura 5 - Distribución de investigaciones educativas

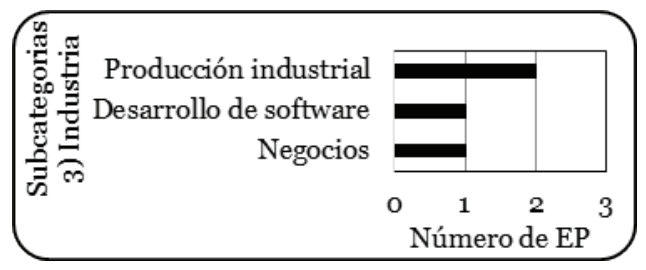

Figura 6 - Distribución de investigaciones en la industria

Respondiendo a la PI2, encontramos que los tres entornos: académico, educación e industria han aplicado elementos de gamificación. Desde una perspectiva similar a la PI1 sólo han sido enfocados en apoyar el cumplimiento de las tareas, por lo que la creación de un equipo de trabajo aun no es abordada en los entornos identificados en este estudio.

Sin embargo, en base a (Jurado et al., 2015), contemplado dentro del entorno industrial, se puede responder indirectamente a la PI2, porque lograron identificar a los miembros de un equipo con ciertas capacidades deseadas para mejorar la comunicación de su conocimiento. También dentro de los EP Chen et al. en (J. Chen et al., 2011) hacen mención a la creación de equipos de estudiantes, donde aplican un método combinado entre: 1) dejar que los estudiantes creen sus propios equipos, 2) crear equipos en base a su desempeño académico previo y 3) crear los equipos de manera aleatoria.

\section{5•3.PI3: ¿Cuáles elementos de gamificación han sido aplicados para mejorar el trabajo en equipo?}

Como respuesta a la PI3 se consideran los elementos descritos en la Figura 1, debido a que han sido aplicados para mejorar el rendimiento de los equipos de trabajo, aunque 
en algunos casos sólo se han logrado mejoras mínimas, demuestran gran potencial para incrementar el rendimiento de los equipos.

\subsection{PI4: ¿Cuáles han sido las principales implementaciones o herramientas para aplicar los elementos de gamificación?}

Respondiendo a la PI4, se muestra en la Figura 7 las principales Implementaciones y Herramientas de Apoyo (IHA), que han sido utilizadas en los estudios con el fin de aplicar los elementos de la gamificación. De acuerdo a los resultados, se han identificado ocho implementaciones y herramientas, distribuidas en las siguientes categorías: el uso de una plataforma web (E. Hernández et al., 2010; Jurado et al., 2015; Knutas, Ikonen, Nikula, et al., 2014; Stanculescu et al., 2016), utilizada en cuatro estudios, los cuales han sido aplicados en sólo un estudio cada uno; método propio (Knutas, Ikonen, Maggiorini, et al., 2014; Korn et al., 2015a, 2015b) y salón de clases, con tres estudios cada uno; Desarrollo de Software (Akpolat \& Slany, 2014; Unkelos-Shpigel \& Hadar, 2015) y una plataforma web tipo Learning Manage System (LMS) (Latulipe et al., 2015; O’Donovan et al., 2013), con dos implementaciones en los estudios; y finalmente, investigación (Bartel et al., 2015), juego serio (Guenaga et al., 2014) y participación en la mejora de procesos de software (MPS) (Dorling \& McCaffery, 2012).

A continuación se describe cómo se realizó la categorización de cada uno de los estudios: plataforma web (IH1) - estudios que implementan los elementos de gamificación mediante el apoyo de una plataforma web, ya sea una existente o creada por los autores; salón de Clases (IH2) - estudios donde los elementos han sido aplicados, principalmente, en el área de la educación de la ingeniería de software; método propio ( $\mathrm{IH}_{3}$ ) - estudios donde el o los autores describen sus propuestas en base a un nuevo método de aplicación, plataforma Web LMS (IH4) - estudios que usan un LMS para aplicar los elementos, principalmente estos estudios se enfocan en áreas de la educación; desarrollo de Software ( $\mathrm{IH}_{5}$ ) - estudios donde se aplican los elementos en las tareas de desarrollo, participación en la MPS (IH6) estudios que usan elementos para fomentar esta actividad, juegos serios ( $\left.\mathrm{IH}_{7}\right)$ - estudios donde se apoyan de un juego serio para aplicar los elementos, y finalmente investigación (IH8) - estudios donde se ha propuesto de forma teórica el uso de los elementos.

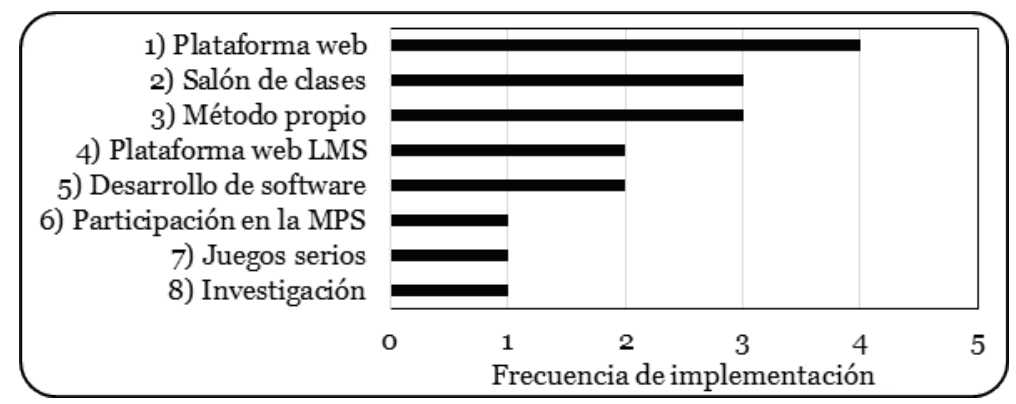

Figura 7 - Implementación y Herramientas de Apoyo.

Cómo se observa, la categoría plataforma Web ha sido una de las opciones más frecuentes en los estudios para aplicar los elementos de gamificación, seguido de la categoría salón de clases también han sido utilizados los elementos, y de la categoría método propio. 
Dentro de la categoría plataforma web, el estudio (Osborne O’Hagan et al., 2014), presenta los elementos de gamificación en una plataforma web para la mejora de procesos de software en las organizaciones, por parte de los equipos de trabajo. Durante su experimento apreciaron que los participantes fueron motivados para participar en la mejora en los procesos de software e incluso mejoraron su rendimiento en las actividades asignadas. Sin embargo, entre los resultados más importantes notaron la ausencia de relajación o diversión mientras los participantes interactuaban con la plataforma.

En la categoría desarrollo de Software, el estudio (Akpolat \& Slany, 2014), presenta cómo la gamificación fue vista como una herramienta viable, porque permitió animar a los estudiantes a participar en los cursos de programación. Sin embargo, un descubrimiento importante en este estudio es la interpretación de la gamificación, nuevamente, como un arma de doble filo, ya que, si la gamificación pierde los aspectos positivos de sus objetivos, esto claramente impactaran de forma negativa en la motivación de los estudiantes. Por lo tanto, se debe tener cuidado en la forma de aplicar las técnicas de gamificación, con el fin de evitar resultados negativos que afecten el público objetivo.

\subsection{PI5: ¿Cuáles son los objetivos a cumplir con el apoyo de los elementos de gamificación?}

Dentro de esta sección se han clasificado los estudios de acuerdo al Objetivo a Cumplir (OC) que persiguen, la Figura 8 muestra el resumen de los objetivos abordados en los estudios. Se han identificado las siguientes categorías: mejorar las habilidades o conocimiento de los estudiantes (OC1) se abordó en seis estudios, (Buisman \& van Eekelen, 2014; Dubois \& Tamburrelli, 2013; Iosup \& Epema, 2014; Knutas, Ikonen, Maggiorini, et al., 2014; Knutas, Ikonen, Nikula, et al., 2014), aplicar gamificación (OC2), se ha llevado a cabo como objetivo en cuatro estudios (Akpolat \& Slany, 2014; Bartel et al., 2015; Korn et al., 2015a, 2015b); mejorar la interacción social (OC3) se ha mencionado en dos estudios (Jurado et al., 2015; Stanculescu et al., 2016); y revisar código (OC4) (Unkelos-Shpigel \& Hadar, 2015), participar en la mejora de procesos de software (OC5) (Dorling \& McCaffery, 2012), motivar a los miembros del equipo (OC6) (E. Hernández et al., 2010), formar equipos de trabajo (OC7) (Latulipe et al., 2015), explicar consecuencias en la toma de decisiones (OC8) (Guenaga et al., 2014), se han mostrado en un sólo estudio cada uno.

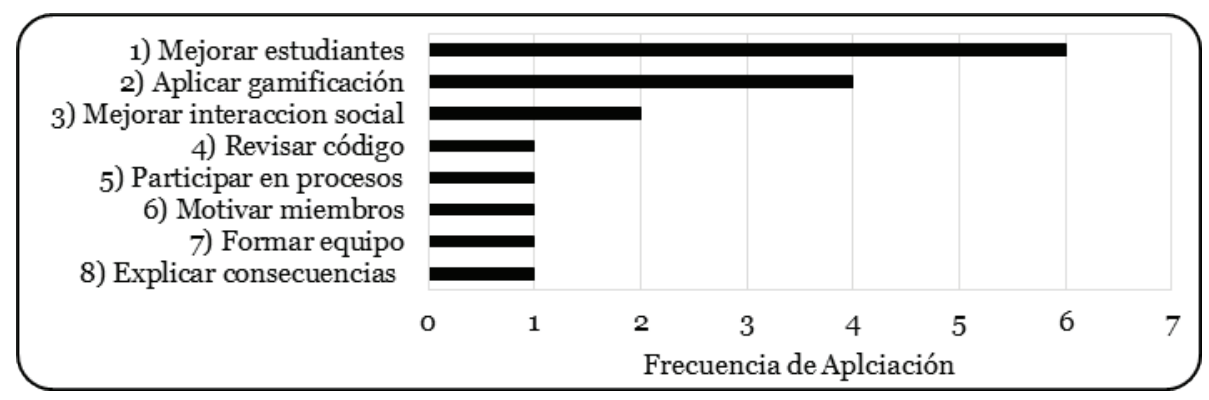

Figura 8 - Objetivos a Cumplir con el Apoyo de Elementos de Gamificación 
Respondiendo a la PI5, los resultados indican que el principal objetivo a cumplir en los estudios ha sido la categoría mejorar las habilidades o conocimientos de los estudiantes, debido a que la mayor parte de los estudios está enfocada en el área de la educación. Uno de los trabajos importantes, para el análisis, dentro de esta categoría fue (Latulipe et al., 2015), debido a que uno de sus objetivos establecidos era la formación de un equipo. Sin embargo, aunque obtuvieron los mismos resultados con y sin la aplicación de los elementos de gamificación, los estudiantes crearon más amigos durante el curso, cumpliendo uno de los propuestos en su estudio.

Por otro lado, el estudio (Jurado et al., 2015) es de relevancia para la investigación, porque uno de sus objetivos se enfoca en mejorar la interacción social de los integrantes del equipo para compartir su conocimiento. Sus conclusiones reflejan buenos resultados de la aplicación de los elementos de gamificación, debido a que no sólo se mejoró compartir el conocimiento, sino además se fortalecieron tres aspectos claves para el trabajo en equipo, que son: la participación, la colaboración y la contribución.

\subsection{Resultados generales}

A continuación, se presentan los resultados generales de la RSL categorizando los estudios por: ubicación geográfica y año de publicación. En la Figura 9 se detalla la distribución de estudios por su ubicación geográfica. Donde se pueden notar cuatro grupos principales por la cantidad de estudios encontrados: Grupo A formado por: Alemania y Estados Unidos (EE. UU.) con cuatro publicaciones, Grupo B formado por: Brasil, España y Países Bajos (NL) con tres publicaciones; Grupo C formado por: China, Finlandia e Irlanda con dos publicaciones; y finalmente el Grupo D formado por: Australia, Austria, Colombia, Israel, Sudáfrica, Taiwán, Turquía y Uruguay con una publicación.

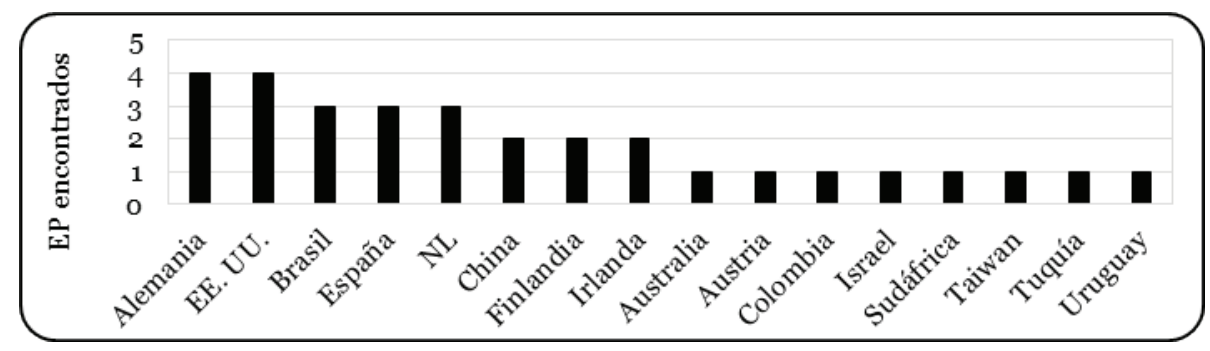

Figura 9 - Distribución de EP por ubicación geográfica

En la Figura 10 se presenta la distribución de EP por año de publicación, se observa que desde los años 2010 - 2013 comenzó débilmente la investigación en temas de gamificación y de trabajo en equipo. Sin embargo, durante los años $2014-2015$ los temas fueron investigados con más frecuencia, demostrando una oportunidad para explorar en diferentes áreas, en este caso para la ingeniería de software.

Dentro del área de la ingeniería de software los elementos de gamificación han sido aplicados en los equipos de trabajo en equipo, principalmente en el entorno educativo, donde se han implementado para equilibrar el conocimiento entrelos integrantes, mejorar 
su comunicación, concluir actividades en tiempo y resolver problemas relacionados con el desarrollo de software. Por otro lado, la gamificación apoya el desempeño personal de los estudiantes por medio de la motivación para: retener información, participar en clases o adquirir nuevos conocimientos, debido a que disfrutan el aprendizaje.

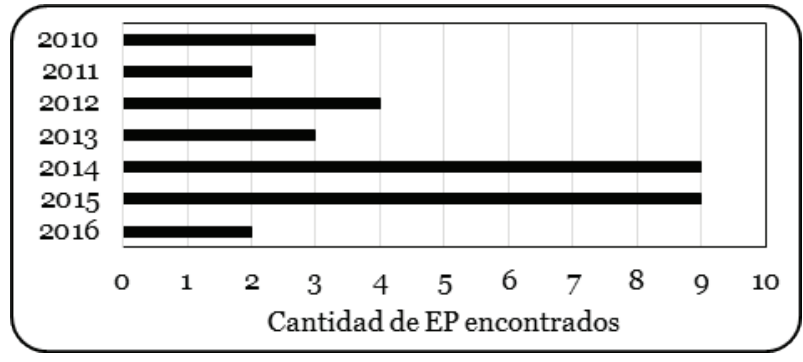

Figura 10 - Distribución de EP por año de publicación

\section{Conclusiones y trabajo futuro}

En el caso de la ingeniería de software, en particular en el entorno industrial en los equipos de desarrollo de software, apenas se está comenzando a explorar la aplicación de la gamificación, debido a la popularidad del tema en los últimos años.

Como resultado de la RSL se ha identificado una carencia del uso de la gamificación para crear un equipo de trabajo, siendo la principal aplicación de gamificación en el cumplimiento de las actividades. Además, se ha logrado la identificación de los elementos de gamificación, los principales factores a tener en cuenta para su implementación y los entornos de aplicación y sus objetivos.

Referente a los elementos de gamificación, se ha identificado que los elementos con mayor frecuencia en su aplicación son: 1) tabla de posiciones, 2) sistemas de puntos, 3) insignias, 4) niveles y 5) barras de progreso.

Con respecto a los factores principales a tener en cuenta para la elección de los elementos adecuados, para implementar la gamificación de acuerdo a los objetivos que se pretendan alcanzar, se han identificado 3 factores: 1) el entorno donde se implementarán, 2) la forma de ser aplicados y 3) el público objetivo.

Es importante mencionar, que se puede aplicar desde un sólo elemento hasta los que sean necesarios para cubrir el o los objetivos propuestos, teniendo en cuenta que el éxito de su implementación está fuertemente relacionado con los tres factores mencionados.

En relación a los entornos de aplicación de los elementos de gamificación, estos se han aplicado principalmente en dos entornos, el entorno de la educación y el entorno industrial. Además, se ha identificado que en ambos entornos la principal herramienta de apoyo para utilizar los elementos de gamificación ha sido una plataforma web. 
En el entorno de la educación se ha hecho uso de los elementos para apoyar el trabajo en equipo en los cursos de ingeniería de software abordando puntos clave, por ejemplo: 1) incrementar la colaboración, 2) mejorar la comunicación, 3) participar e interactuar efectivamente, 4) incrementar la activada social en clases, 5) cumplir con las actividades del curso y 6) incentivar el trabajo en equipo. En este contexto se han aplicado los elementos de gamificación para mejorar el rendimiento académico de los estudiantes.

En el entorno industrial, se encontraron pocos estudios en relación al tema de gamificación y trabajo en equipo, en los cuales la aplicación de los elementos tiene diferentes objetivos como: 1) cumplir las actividades asignadas, 2) crear, refinar y compartir el conocimiento para el aprendizaje, 3) mejorar la participación, colaboración y contribución de los trabajadores en el desarrollo de software y 4) incrementar el comportamiento de la interacción social de los trabajadores.

La aplicación los elementos de gamificación puede variar dependiendo de las metas establecidas. Sin embargo, se puede emplear desde uno hasta los elementos que sean necesarios para cumplir las necesidades, demostrando que la combinación de dos o más elementos puede lograr los resultados esperados, siempre y cuando se tengan en cuenta los 3 factores previamente mencionados. De la misma forma, el uso de las herramientas de apoyo puede variar dependiendo de los elementos utilizados.

Finalmente, los elementos de gamificación pueden ser aplicados en los equipos para: 1) mejorar las competencias o habilidades de sus integrantes durante el desarrollo de un proyecto de software; 2) identificar modelos de personalidades y de esta forma ser aplicados para obtener la combinación más eficaz para integrar un equipo de trabajo; y 3) motivar a los integrantes de un equipo para cumplir los objetivos de su trabajo, con la motivación adecuada en actividades de colaboración o comunicación incluso se podría mejorar el nivel de cohesión del equipo.

Como trabajo futuro, se están desarrollando ambientes enfocados en la adecuada creación de equipos mediante la combinación de sus habilidades, conocimientos y estilos interactivos, con el objetivo de reducir el tiempo de integración entre los miembros del equipo, y de esta manera lograr un equipo altamente efectivo.

\section{Referencias}

Akpolat, B. S., \& Slany, W. (2014). Enhancing software engineering student team engagement in a high-intensity extreme programming course using gamification. 2014 IEEE 27th Conference on Software Engineering Education and Training, CSEE and T 2014 - Proceedings, 149-153. https://doi.org/10.1109/CSEET.2014.6816792

Bartel, A., Figas, P., \& Hagel, G. (2015). Towards a Competency-based Education with Gamification Design Elements. Proceedings of the 2015 Annual Symposium on Computer-Human Interaction in Play - CHI PLAY '15, 457-462. https://doi. org/10.1145/2793107.2810325

Bei, J. L. (2013). The selection of incentive team-based reward model. International Conference on Management Science and Engineering - Annual Conference Proceedings, 987-992. https://doi.org/10.1109/ICMSE.2013.6586398 
Berkling, K. (2016). Gamification Behind the Scenes Designing a Software Engineering Course. Designing Games for Improving the Software Development Process. In Communications in Computer and Information Science (Vol. 583, pp. 274-292). https://doi.org/10.1007/978-3-319-29585-5

Buisman, A. L. D., \& van Eekelen, M. C. J. D. (2014). Gamification in educational software development. Proceedings of the Computer Science Education Research Conference on - CSERC '14, VV, 9-20. https://doi.org/10.1145/2691352.2691353

Chen, J., Qiu, G., Yuan, L., Zhang, L., \& Lu, G. (2011). Assessing Teamwork Performance in Software Engineering Education: A Case in a Software Engineering Undergraduate Course. 2011 18th Asia-Pacific Software Engineering Conference, 17-24. https://doi.org/10.1109/APSEC.2011.50

Chen, P. C., Chern, C. C., \& Chen, C. Y. (2012). Software project team characteristics and team performance: Team motivation as a moderator. Proceedings - Asia-Pacific Software Engineering Conference, APSEC, 1, 565-570. https://doi.org/10.1109/APSEC.2012.152

De O. Melo, C., Santana, C., \& Kon, F. (2012). Developers motivation in agile teams. Proceedings - 38th EUROMICRO Conference on Software Engineering and Advanced Applications, SEAA 2012, 376-383. https://doi.org/10.1109/SEAA.2012.45

Dorling, A., \& McCaffery, F. (2012). The gamification of SPICE. Communications in Computer and Information Science, 290 CCIS, 295-301. https://doi. org/10.1007/978-3-642-30439-2_35

Dubois, D. J., \& Tamburrelli, G. (2013). Understanding gamification mechanisms for software development. Proceedings of the 2013 9th Joint Meeting on Foundations of Software Engineering, 659. https://doi.org/10.1145/2491411.2494589

Dutra, A. C. S., Prikladnicki, R., \& Franca, C. (2015). What Do We Know about High Performance Teams in Software Engineering? Results from a Systematic Literature Review. 2015 41st Euromicro Conference on Software Engineering and Advanced Applications, 183-190. https://doi.org/10.1109/SEAA.2015.24

Estacio, B., Prikladnicki, R., Mora, M., Notari, G., Caroli, P., \& Olchik, A. (2014). Software kaizen: Using agile to form high-perfomance software development teams. Proceedings - 2014 Agile Conference, AGILE 2014, 1-10. https://doi.org/10.1109/ AGILE.2014.10

Gasca-Hurtado, G. P., Peña, A., Gómez-Álvarez, M. C., Plascencia-Osuna, Ó. A., \& CalvoManzano, J. A. (2015). Realidad virtual como buena práctica para trabajo en equipo con estudiantes de ingeniería. RISTI - Revista Iberica de Sistemas E Tecnologias de Informacao, 2015(16), 76-91. https://doi.org/10.17013/risti.16.76-91

Guenaga, M., Eguiluz, A., Rayon, A., Nunez, A., \& Quevedo, E. (2014). A serious game to develop and assess teamwork competency. 2014 International Symposium on Computers in Education, SIIE 2014, 183-188. https://doi.org/10.1109/ SIIE.2014.7017727

H. Gaynor, G. (2015). Identifying Team Members. IEEE Engineering Management Review, 43(4), 7-9. Retrieved from http://dx.doi.org/10.1109/EMR.2015.7433677 
Hernández, E., Colomo Palacios, R., \& deAmescua Seco,A. (2010). Gamiware: A Gamification Platform for Software Process Improvement. Communications in Computer and Information Science, 99, 233-244. https://doi.org/10.1007/978-3-642-15666-3

Hernández, L., Muñoz, M., Mejia, J., \& Peña, A. (2016). Gamificación en equipos de trabajo en la ingeniería de software: Una revisión sistemática de la literatura Gamification in software engineering teamworks : A systematic literature review. International Conference on Software Process Improvement (CIMPS), 5, 1-8. https://doi.org/10.1109/CIMPS.2016.7802799

Iosup, A., \& Epema, D. (2014). An experience report on using gamification in technical higher education. Proceedings of the 45th ACM Technical Symposium on Computer Science Education - SIGCSE '14, (2008), 27-32. https://doi. org/10.1145/2538862.2538899

Jovanovic, M., Mesquida, A.-L., \& Antònia, M. (2010). Process Improvement with Retrospective Gaming in Agile Software Development. Communications in Computer and Information Science, 99, 233-244. https://doi.org/10.1007/978-3642-15666-3

Jurado, J.L., Fernandez,A., \& Collazos, A.C. (2015).Applying gamification in the context of knowledge management. 15th International Conference on Knowledge Technologies and Data-Driven Business, 1, 10-13. https://doi.org/10.1145/2809563.2809606

Kitchenham, B., \& Charters, S. (2007). Guidelines for performing Systematic Literature reviews in Software Engineering. Engineering, 45(4ve), 1051. https://doi. org/10.1145/1134285.1134500

Knutas, A., Ikonen, J., Maggiorini, D., Ripamonti, L., \& Porras, J. (2014). Creating Software Engineering Student Interaction Profiles for Discovering Gamification Approaches to Improve Collaboration. International Conference on Computer Systems and Technologies - CompSysTech'14, 378-385. https://doi. org/10.1145/2659532.2659612

Knutas, A., Ikonen, J., Nikula, U., \& Porras, J. (2014). Increasing collaborative communications in a programming course with gamification. Proceedings of the $15^{\text {th }}$ International Conference on Computer Systems and Technologies - CompSysTech '14, 883, 370-377. https://doi.org/10.1145/2659532.2659620

Korn, O., Funk, M., \& Schmidt, A. (2015a). Design approaches for the gamification of production environments. Proceedings of the 8th ACM International Conference on PErvasive Technologies Related to Assistive Environments - PETRA '15, 1-7. https://doi.org/10.1145/2769493.2769549

Korn, O., Funk, M., \& Schmidt, A. (2015b). Towards a Gamification of Industrial Production. A Comparative Study in Sheltered Work Environments, (JUNE), 8493. https://doi.org/10.1145/2774225.2774834

Kosa, M., \& Yilmaz, M. (2010). Designing Games for Improving the Software Development Process. Systems, Software and Services Process Improvement. Communications in Computer and Information Science, 99, 233-244. https://doi.org/10.1007/978-3642-15666-3 
Latulipe, C., Long, N. B., \& Seminario, C. E. (2015). Structuring Flipped Classes with Lightweight Teams and Gamification. Proceedings of the 46th ACM Technical Symposium on Computer Science Education, 392-397. https://doi. org/10.1145/2676723.2677240

Lynch, T. D., Herold, M., Bolinger, J., Deshpande, S., Bihari, T., Ramanathan, J., \& Ramnath, R. (2011). An agile boot camp: Using a LEGOß-based active game to ground agile development principles. Proceedings - Frontiers in Education Conference, FIE, 1-6. https://doi.org/10.1109/FIE.2011.6142849

M2 Research. (2016). M2 Research - Gamification. Retrieved June 8, 2016, from http:// m2research.com/Gamification.htm

Matturro, G., Raschetti, F., \& Fontán, C. (2015). Soft skills in software development teams: A survey of the points of view of team leaders and team members. Proceedings - 8th International Workshop on Cooperative and Human Aspects of Software Engineering, CHASE 2015, 101-104. https://doi.org/10.1109/CHASE.2015.30

O’Donovan, S., Gain, J., \& Marais, P. (2013). A case study in the gamification of a university-level games development course. Proceedings of the South African Institute for Computer Scientists and Information Technologists Conference on SAICSIT '13, 242-251. https://doi.org/10.1145/2513456.2513469

Osborne O’Hagan, A., Coleman, G., \& O’Connor, R. V. (2014). Software Development Processes for Games: A Systematic Literature Review, 425(June), 182-193. https:// doi.org/10.1007/978-3-662-43896-1_16

Poy-Castro, R., Mendaña-Cuervo, C., \& González, B. (2015). Diseño y evaluación de un juego serio para la formación de estudiantes universitarios en habilidades de trabajo en equipo. RISTI - Revista Ibérica de Sistemas E Tecnologias de Informação, (E3), 71-83. https://doi.org/10.17013/risti.e3.71-83

Stanculescu, L. C., Bozzon, A., Sips, R.-J., \& Houben, G. (2016). Work and Play: An Experiment in Enterprise Gamification. Proceedings of the 19th ACM Conference on Computer-Supported Cooperative Work \& Social Computing - CSCW '16, 345357. https://doi.org/10.1145/2818048.2820061

Teh, A., Baniassad, E., Van Rooy, D., \& Boughton, C. (2012). Social psychology and software teams: Establishing task-effective group norms. IEEE Software, 29(4), 53-58. https://doi.org/10.1109/MS.2011.157

Unkelos-Shpigel, N., \& Hadar, I. (2015). Gamifying software engineering tasks based on cognitive principles: The case of code review. Proceedings - 8th International Workshop on Cooperative and Human Aspects of Software Engineering, CHASE 2015, 119-120. https://doi.org/10.1109/CHASE.2015.21

Vasconcelos, J. B., Kimble, C., Carreteiro, P., \& Rocha, Á. (2017). The application of knowledge management to software evolution. International Journal of Information Management, 37(1), 1499-1506. http://doi.org/10.1016/j.ijinfomgt.2016.05.005 